\title{
Percutaneous Transforaminal Endoscopic Decompression for Lumbar Foraminal Stenosis
}

\section{Young-Ha Woo*, Heung-Tae Jung, In-Bo Kim, Woo-Seong Sun and Dong-Wook Jung}

Department of Orthopaedic Surgery, Bumin Busan Hospital, Busan, Republic of Korea

*Corresponding author: Young-Ha Woo, Department of Orthopedic Surgery, Busan Bumin Hospital, Busan, Republic of Korea, Tel: 051-330-3082; Fax: 051-330-3082; Email: woo0ha@naver.com

Received date: October 23, 2017; Accepted date: November 01, 2017; Published date: November 06, 2017

Citation: Woo YH, Jung HT, Kim IB, Sun WS, Jung DW (2017) Percutaneous Transforaminal Endoscopic Decompression for Lumbar Foraminal Stenosis. J Clin Exp Orthop Vol.3 No.3: 42

Copyright: (C2017 Woo YH, et al. This is an open-access article distributed under the terms of the Creative Commons Attribution License, which permits unrestricted use, distribution, and reproduction in any medium, provided the original author and source are credited.

\section{Abstract}

Background: Several different techniques exist to address the pain and disability caused by isolated nerve root impingement. Failure to adequately decompress the lumbar foramen may lead to failed back surgery syndrome. However, aggressive treatment often causes spinal instability or may require fusion for satisfactory results. We describe a novel technique for decompression of the lumbar nerve root and demonstrate its effectiveness in relief of radicular symptoms.

Materials and methods: Percutaneous transforaminal endoscopic decompression was performed by removal of the herniated disc in patients with lumbar foraminal stenosis. 91 patients underwent the procedure from 2003 to 2015. Those who demonstrated neurogenic claudication without spinal instability or central canal stenosis and failed conservative management were eligible for the procedure. These patients were followed for an average of 39 months to evaluate outcomes.

Results: The results were excellent in 50 patients, good in 39 , and fair in 2. There were no surgery-related complications. Lateral lumbar spine flexion-extension radiography was performed in all patients at various times ranging from 3 to 48 months after the surgery, and no evidence of postoperative spinal instability was found.

Conclusions: Percutaneous transforaminal endoscopic decompression is an effective means to decompress the lumbar nerve root foramen without causing spinal instability.

\section{Keywords: $\quad$ Foraminal stenosis; Percutaneous} transforaminal endoscopic decompression endoscopy; Surgery; Disc herniation

\section{Introduction}

Lumbar foraminal stenosis is a common cause of radiculopathy in lower back pain and is a common disease that occurs in $8-11 \%$ after lumbar degenerative diseases surgery [1]. In the case of foraminal stenosis with spondylosis, it makes the upper articular surface hypertrophy, buckling of the ligamentum flavum, disc protrusion, the fibrous ring protrusion, and formation of osteophyte. These changes may result in impingement of the exiting nerve root $[2,3]$. In addition, a reduction in the height of the intervertebral disc causes stenosis of the nerve foraman and often causes the lumbar spinal cord syndrome. Non-perceived neurological impairment because of foraminal stenosis or the incomplete treatment of foraminal stenosis has been associated with the cause of Failed Back Surgery Syndrome [4]. In a study of Failed Back Surgery Syndrome after spinal surgery by Burton, it was attributed to lack of cognition and incomplete treatment of formanial stenosis, which was considered to be the cause of pain in nearly $60 \%$ of patients after surgery. Thus, lumbar foraminal stenosis is an important pathological feature for identifying patients treated with radiating pain [4]. The ideal surgical treatment for relieving pressure on the exiting nerve root for lumbar foraminal stenosis has not yet been established. Microscopic diskectomy described by Williams so far has been the criterion standard of surgical treatment of lumbar disc herniation [5].

There are other methods of decompressing foraman pressure such as foraminotomy, facetectomy, partial pediculectomy, fusion, distraction instrumentation, and posterior lumbar interbody fusion. However, the authors believe that complete removal of the posterior joint may cause spinal instability and occasionally require posterior fusion.

After arthroscopic removal of the disc herniation was introduced, various techniques have been introduced in cases of mild disc herniations [6,7]. Despite being a suitable method, percutaneous endoscopic discectomy through foramen is contraindication for limited surgical visibility [8-11].

The development of endoscopic instruments and techniques allows discectomy through the use of direct visualization of pathologic lesions and nerve structures [10,12-14].

The purpose of this study was to report the results of a percutaneous endoscopic decompression in patients with lumbar foraminal stenosis. 


\section{Materials and Methods}

Surgery for patients with 1 or 2 level neuromuscular disease caused by lumbar stenosis or difficult to treat with conservative treatment was performed in 91 patients from March 2003 to October 2015. Patients with disc herniation were excluded from the study

The patients consisted of 32 men and 59 women, ranging in age from 46 to 83 years (mean age 67 years). The most common nerve root was L5 ( 45 patients), followed by L4 ( 29 patients), L3 patients (10 patients), and L2 patients ( 7 patients). Patients having neuromuscular disease in two levels were 34 cases (37.4\%). Back pain was also present in 38 patients. 27 Patients having myelopathy were observed and 13 patients experienced abnormal reflexes.

Diagnosis was performed by $\mathrm{CT}$ and $\mathrm{MRI}$ in all patients. Reconstructed $\mathrm{CT}$ included the presence of a bony spur extending from the posterior lateral vertebral body, the articular surface to intervertebral foramen in the findings suggestive of stenosis.

The follow-up period ranged from 3 to 148 months (mean 39 months)

\section{Surgical technique}

We used the Hopkins (R) Telescope $25^{\circ}$ from Storz and the UNIDRIVE (R) S III NEURO SCB with endoscopic drill. Before surgery, 3D CT is used to confirm the area of the nerve root impairment (Figure 1). Then a preliminary plan by drawing a hypothetical line (Figure 2). An x-ray is taken to confirm the exact location of the incision and the area of the stenosis where the exiting root is pressed is marked with a spinal needle and Indigo Carmine solution. The upper and lower laminae adjacent to the facet joint are then exposed by paramedian horizontal incision. After exposing posterior joint, we explore upper articular part by curved instrument (Figure 3), and drill superiolateral part of posterior articular and superiolateral margin of interarticular part by a high-speed drill and a rongeur (Figure 4). At this time, only the superior articular process of the caudal vertebral body is partially removed, and the removal site of the posterior joint should not exceed half of the total posterior joint. The intertransverse ligaments are then removed to expose the nerve roots in lateral foramen. The affected nerve roots follow the neuroforamen and by standard interlaminar approach, medial part of foramen decompression is completed (Figure 5). In order to confirm that the decompression is sufficient, small nerve hook is used to carefully identify. The nerve roots can be decompressed by removing the superolateral portion of the facet joint and the upper lateral margin of the interarticularis. Through endoscopy, after an exting root is confirmed, enough decompression from preganglionic to postganglionic is performed and the operation is completed (Figures 6 and 7).

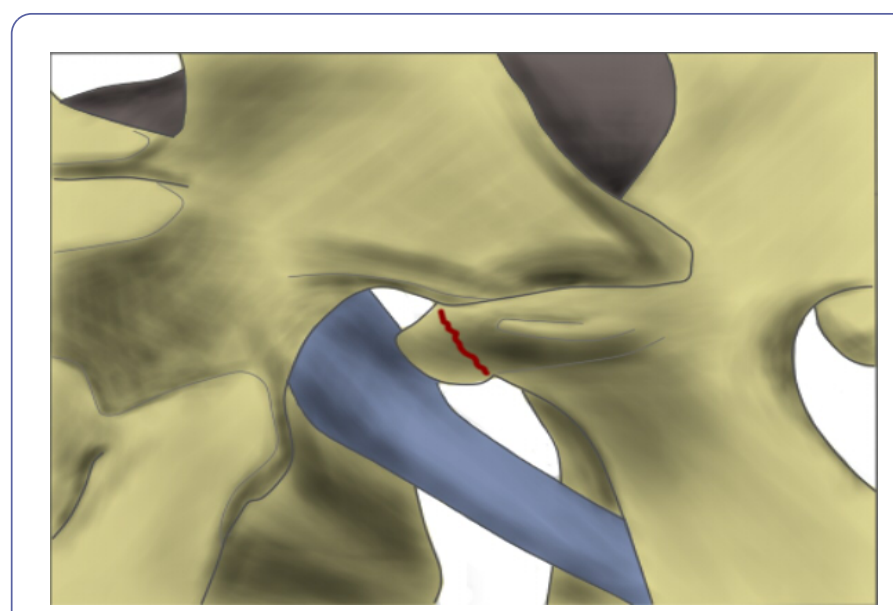

Figure 1: The borders of the foraminal space can be identified.

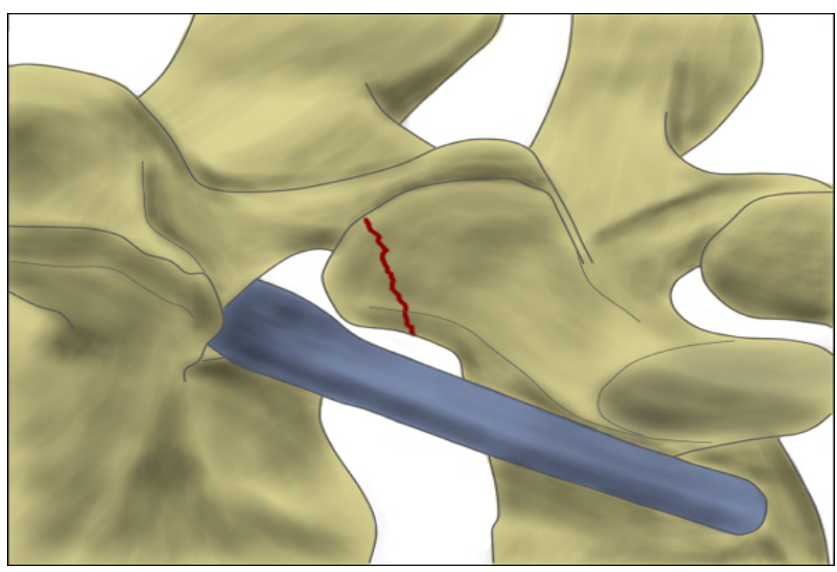

Figure 2: Planned partial resection of the upgoing facet.

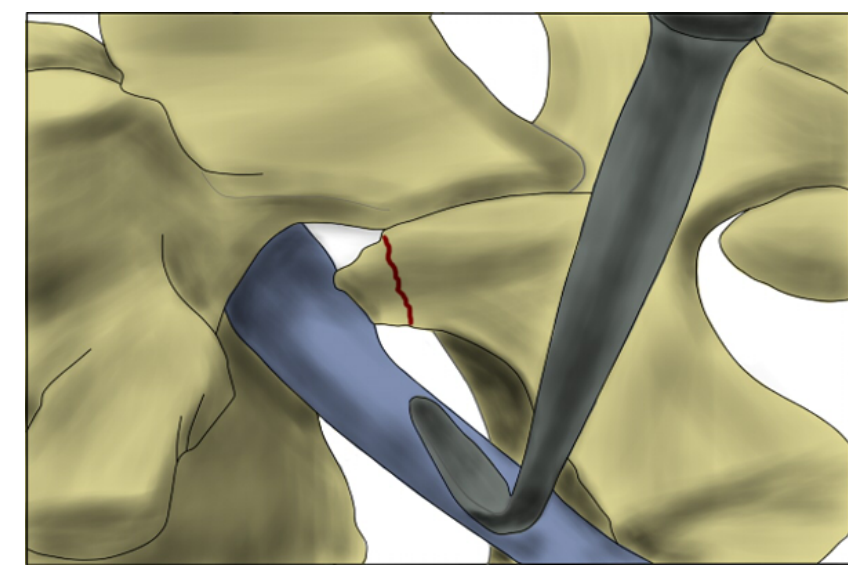

Figure 3: Curved instrument inserted to palpate the pedicle before osteotomy. 


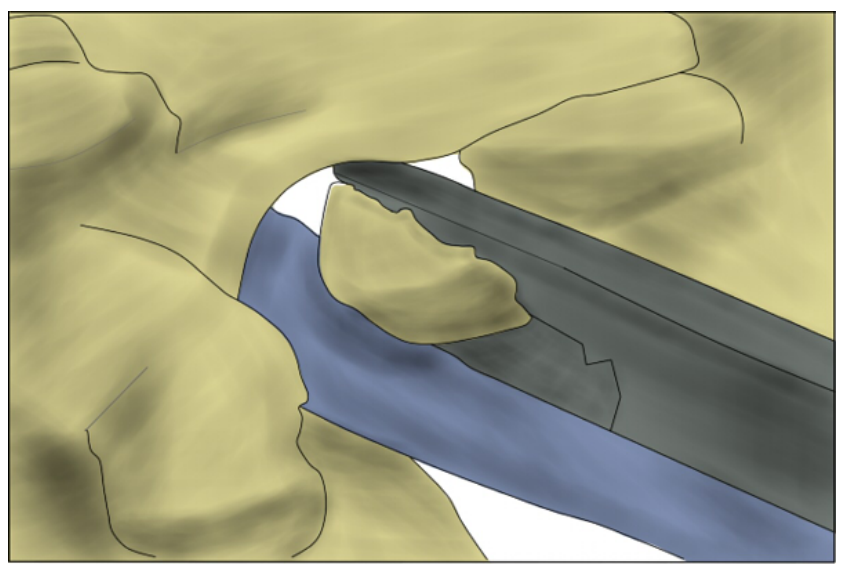

Figure 4: Osteotome performing the lumbar partial facetectomy.

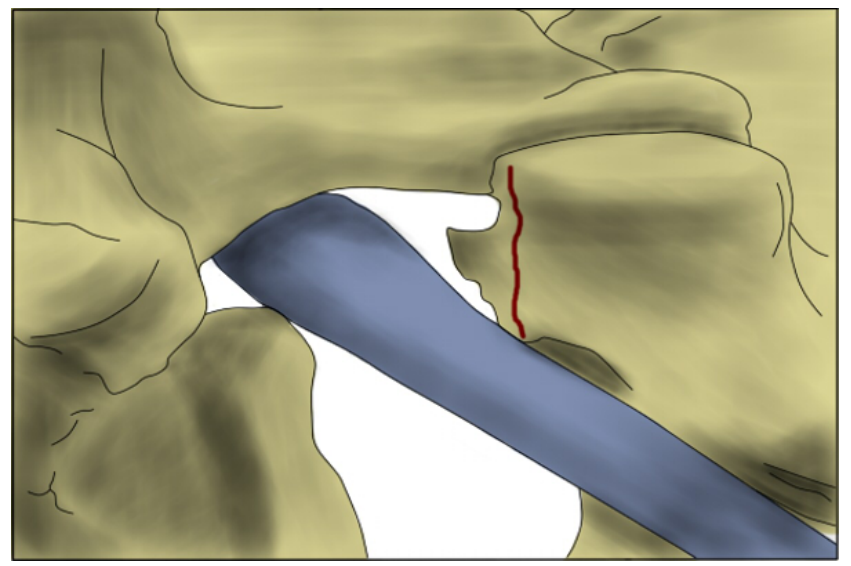

Figure 5: Neuroforamen after resection of bone.

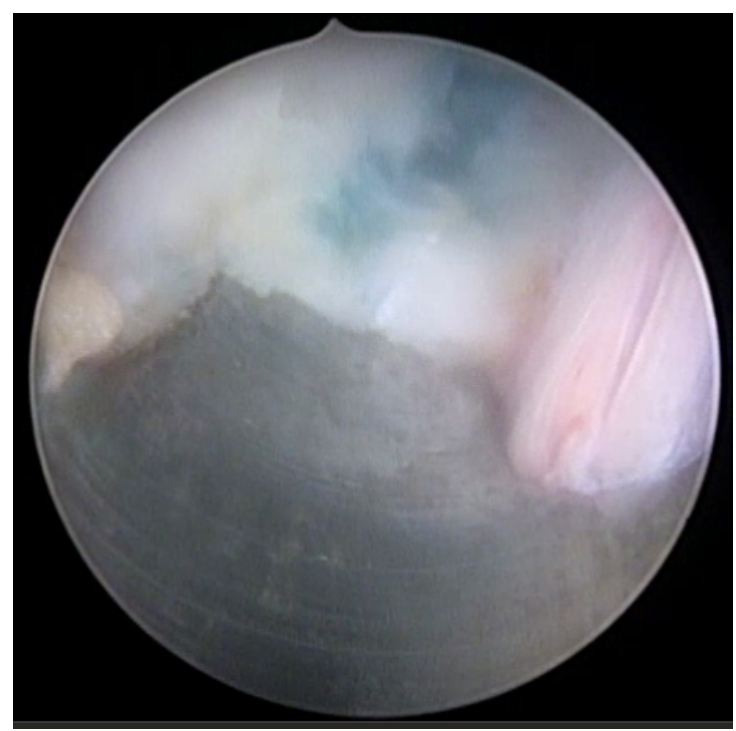

Figure 6: Nerve root identified and squeezing technique was done.

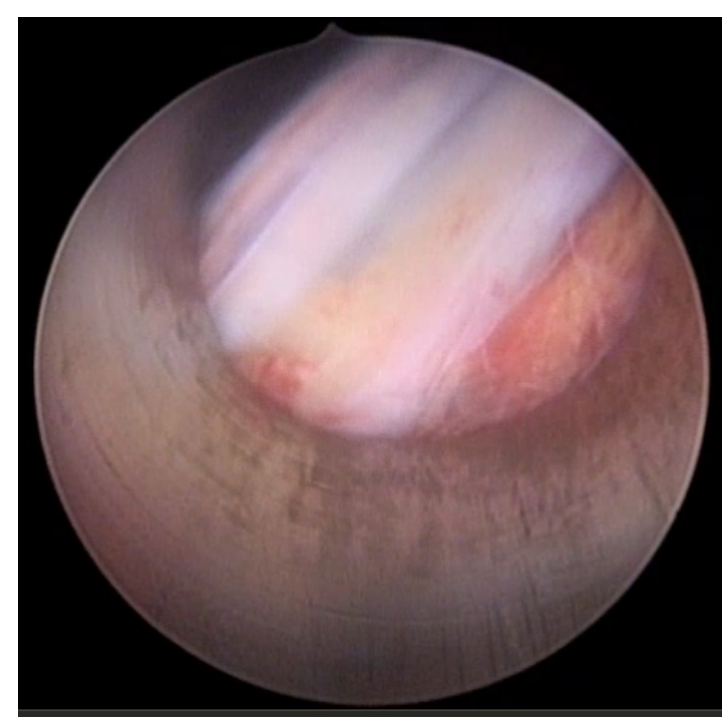

Figure 7: Decompression of the nerve root identified.

\section{Results}

The results were defined as Excellent (no pain), good (fairly low back pain), fair (moderate neuropathy), and poor (unchanged or deep) according to the Macnab classification. The results were excellent in 50 patients, good in 39 patients and fair in 2 patients. There were no surgical complications. The lateral lumbar flexion-extension radiographs were performed on all patients from 3 months to 48 months postoperatively, and no evidence of postoperative lumbar instability was found. Postoperative MRI showed preservation of the major part of the lumbar facet joint, and osteophyte and intervertebral discs that were observed in the foramen disappeared which meant sufficient decompression (Figure 8).
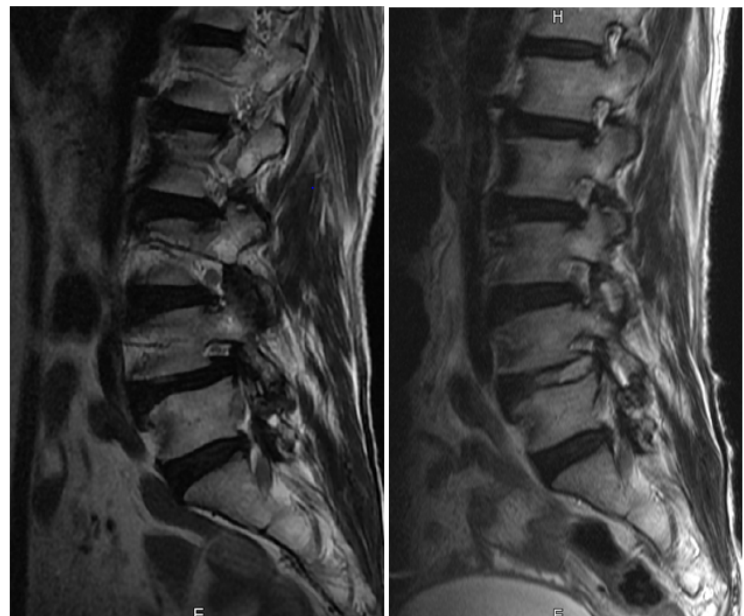

Figure 8: Preoperative and postoperative magnetic resonance imaging showing the removed disc material and decompressed nerve root. 


\section{Discussion}

Spinal stenosis is a broad term that encompasses all the entities that reduce the space of the spinal cord in the vertebral canal. According to Postacchini [15], degenerative lumbar spine stenosis consists of 3 types. Central stenosis means stenosis of the central part of the canal. Lateral stenosis involves the path of the nerve bundle from the thecal sac to the entrance of the intervertebral foramen. Foraminal stenosis is caused by the narrowing of the neuroforamen. The choice of treatment and surgical intervention depends on the pathologic location and must be considered for satisfactory outcomes. Jenis and An [1] described pathological $q$ of the intervertebral stenosis.

Lumbar spondylosis is caused by loss of disc height and anterior and upper migration of the superior facet. The anteroposterior dimension of the neuroforamen decreases with decreasing height between the spinal discs. In addition, hypertrophy of the ligamentum flavum and osteophyte formation exacerbates compression. The two craniocaudal dimensions may be damaged by the bulging annulus fibrosus or the endplate osteophyte of the herniated disc. The sum of the degenerative changes mentioned above can lead to a mild narrowing of the exiting space of the nerve bundle that can potentially cause back pain and radicular symptoms. We tried to prove meaningful results by partial facetectomy in intervertebral stenosis.

Several surgical approaches can use to reach the extraforaminal lateral region of the lumbar spine $[16,17]$. Some authors advocated complete removal of the posterior joints for decompressing of the spinal canal and exploration of the intervertebral foramen. However, these factors may cause spinal instability and may occasionally require posterior fusion later $[16,18,19]$. Abdullah et al. [20], stated they removed the medial part of the facet in their study and drilled the lateral part. The disadvantage of the medial subtotal facetectomy is that it is difficult to visualize location of lesion and that decompression of the lateral foramen may be incomplete. Reulen et al. [21], showed that in the most of cases, almost the inferior articular process in superior vertebral body should be removed for decompression of the nerve root. Removing these bones can weaken the articular process floor, which can lead to fractures and post-operative spinal instability [19].

Clinical studies of intra and extraforaminal neuromuscular decompression have shown that patients with poor clinical status have undergone total facetectomy or have been removed pars interarticularis without spinal fusion [22]. Some authors have emphasized that spinal fusion is essential even if total facetectomy is required [1,16,22]. Epstein [16], also insists that primary spinal fusion should be considered in patients undergoing total facetectomy. Total facetectomy, without severe and apparent vertebral instability, results in a modified path in the three column of vertabrae, which can result in severe postoperative back pain due to degeneration of adjacent discoligamentous structures $[19,23]$. Although fracture of the facet joint does not cause acute instability, it may change the load on adjacent discs and accelerate degeneration [19].
According to Kunogi and Hasue, the spinal fusion procedure was essential in $2(25 \%)$ of 8 patients with disc herniation after total facetectomy [22]. The facet joint is considered to be critical to maintaining stability of rotation.

The advantage of the surgical technique described by the authors is that they can preserve the stability of the spine. Most of the stability of the lumbar spine comes from the anterior annulus fibrosus as well as the anterior longitudinal ligament.

As the original instability is ruled out, partial facetectomy can be performed safely without the need for fusion or instrumentation. The surgical techniques described by the authors provide a good approach by making nerve roots visualization, conservation of the major part of the facet joint. Improved visualization minimizes the risk of nerve root damage to the lateral and medial part of the intervertebral foramen. Nerve roots can be decompressed by removing the superolateral portion of the facet joint and the superolateral margin of the interarticularis. This preservation of functional facet joints reduces the possibility of postoperative instability. Even for the authors of this study, the clinical results of this surgical technique were excellent in most cases.

Therefore, the percutaneous endoscopic transforaminal decompression in the lumbar spine may be the standard approach for lumbar foraminal stenosis, it is a safe and effective surgical procedure that does not cause secondary vertebral instability after surgery, and it shows an improvement of symptoms through long term follow-up.

\section{Conclusion}

The authors' technique is safe, minimally invasive, provides excellent visualization of the lateral vertebral structure, and requires minimal bone resection, thus avoiding the risk of secondary instability due to total facetectomy.

\section{Conflicts of Interest}

We have been financed by a certain amount of money from the storz company.

\section{References}

1. Jenis LG, An HS (2000) Spine update: lumbar foraminal stenosis. Spine 25: 389-94.

2. Hasegawa T, An HS, Haughton VM, Nowicki BH (1995) Lumbar foraminal stenosis: critical heights of the intervertebral discs and foramina. A cryomicrotome study in cadavera. J Bone Joint Surg 77: $32-8$.

3. Shenouda E, Gill S (2002) Laminal fenestration for the treatment of lumbar nerve root foraminal stenosis. Br J Neurosurg 16: 494-6.

4. Burton CV, Kirkaldy-Willis W, Yong-Hing K, Heithoff KB (1981) Causes of failure of surgery on the lumbar spine. Clin Orthop Relat Res 157: 1919.

5. Williams RW (1978) Microlumbar discectomy: a conservative surgical approach to the virgin herniated lumbar disc. Spine 3: 175-82. 
6. Kafadar A, Kahraman S, Akboru M (2006) Percutaneous endoscopic transforaminal lumbar discectomy: a critical appraisal. Minim Invasive Neurosurg 49: 74-9.

7. Kambin P, O'Brien E, Zhou L, Schaffer JL (1998) Arthroscopic microdiscectomy and selective fragmentectomy. Clin Orthop Relat Res 347: 150-67.

8. Ditsworth DA (1998) Endoscopic transforaminal lumbar discectomy and reconfiguration: a postero-lateral approach into the spinal canal. Surg Neurol 49: 588-97.

9. Hermantin FU, Peters T, Quartararo L, Kambin P (1999) A prospective, randomized study comparing the results of open discectomy with those of video-assisted arthroscopic microdiscectomy. J Bone Joint Surg Am Jul 81: 958-65.

10. Mathews HH (1996) Transforaminal endoscopic microdiscectomy. Neurosurg Clin N Am 7: 59-63.

11. Mayer HM, Brock M (1993) Percutaneous endoscopic discectomy: surgical technique and preliminary results compared to microsurgical discectomy. J Neurosurg 78: 216-25.

12. Yeung AT, Yeung CA (2003) Advances in endoscopic disc and spine surgery: foraminal approach. Surg Technol Int 11: 255-63.

13. Yeung AT (2000) The evolution of percutaneous spinal endoscopy and discectomy: state of the art. Mt Sinai J Med 67: 327-32.

14. Yeung AT, Tsou PM (2002) Posterolateral endoscopic excision for lumbar disc herniation: Surgical technique, outcome, and complications in 307 consecutive cases. Spine 27: 722-31.

15. Postacchini $F$ (1999) Surgical management of lumbar spinal stenosis. Spine 24: 1043-7.
16. Epstein NE (1995) Evaluation of varied surgical approaches used in the management of 170 far-lateral lumbar disc herniations: indications and results. J Neurosurg 83: 648-56.

17. Hassler W, Brandner S, Slansky I (1996) Microsurgical management of lateral lumbar disc herniations: combined lateral and interlaminar approach. Acta Neurochir 138: 907-11.

18. Garrido E, Connaughton PN (1991) Unilateral facetectomy approach for lateral lumbar disc herniation. J Neurosurg 74: 754-6.

19. Haher TR, O'Brien M, Dryer JW, Nucci R, Zipnick R, et al. (1994) The Role of the Lumbar Facet Joints in Spinal Stability: Identification of Alternative Paths of Loading. Spine 19: 2667-70.

20. Abdullah AF, Wolber PG, Warfield JR, Gunadi KI (1988) Surgical management of extreme lateral lumbar disc herniations: review of 138 cases. Neurosurg 22: 648-53.

21. Reulen H-J, Müller A, Ebeling U (1996) Microsurgical anatomy of the lateral approach to extraforaminal lumbar disc herniations. Neurosurg 39: 345-51.

22. Kunogi J-i, Hasue M (1991) Diagnosis and operative treatment of intraforaminal and extraforaminal nerve root compression. Spine 16: 1312-20.

23. Porchet F, Chollet-Bornand A, de Tribolet N (1999) Long-term follow up of patients surgically treated by the far-lateral approach for foraminal and extraforaminal lumbar disc herniations. J Neurosurg Spine 90: 59-66. 